\title{
The Structure of Plant-Animal Mutualistic Networks
}

\author{
Jordi Bascompte \\ Pedro Jordano
}

Plant-animal mutualistic networks can be described as bipartite graphs depicting the interactions between two distinct sets: plants and animals. These mutualistic networks have been found to be highly structured. Specifically, they show a nested pattern in which specialists interact with proper subsets of the species generalists interact with. This pattern is important for understanding coevolution in species-rich communities which can be reduced neither to pairs of coevolving species nor to diffuse, randomly-interacting assemblages. We discuss the dynamic implications of network structure from the points of view of coevolution, community ecology, and conservation biology.

\section{INTRODUCTION}

Studies on community organization have largely focussed on a small subset of species interactions: trophic relationships depicted in traditional food webs. In order to improve our understanding of community organization, we need to ex-

Bascompte, J. and Jordano, P. 2006. The structure of plant-animal mutualistic networks. Pages 143-159 in Pascual, M. and Dunne, J. (eds.). Ecological networks: linking structure to dynamics in food webs. Oxford University Press, Oxford, UK. 
plore how other types of interactions shape communities. In this chapter we consider a very important and largely unexplored interaction at the community level: plant-animal mutualisms. It is well accepted that the interactions between plants and the animals that pollinate them or disperse their fruits have played a major role in the generation of terrestrial biodiversity (Ehrlich and Raven 1964). For example, the life cycle of $89.5 \%$ of the woody plants in tropical rainforests depend on vertebrate animals for seed dispersal (Jordano 2000). Similarly, it is well accepted that the elimination of pollinators or seed dispersers would have catastrophic consequences within decades, with the elimination of much of terrestrial biodiversity (Thompson 2002, Koh et al. 2004).

Lots of studies on mutualisms have focused on very specialized interactions between a pair of species. This partly arises because of the attraction towards text-book examples of highly specific interactions such as the ones between orchids whose flowers perfectly mimic the female of the insect species that pollinate them. This has originated a large body of work on pair-wise coevolution. However, when one looks at nature, mutualistic interactions oftentimes involve much larger sets of species. The alternative to one-to-one coevolution up to the recent years has been the concept of diffuse coevolution (Janzen 1980). This concept assumes that interactions are not species-to-species, but guild-to-guild. These interactions are very variable from year to year and also through space (Herrera 1988). Since a species is subjected to highly variable and unpredictable selection pressures, diffuse coevolution would thus preclude fine tuned adaptations. This has been a main argument to question pair-wise coevolution as a prevalent mechanism explaining highly-diversified mutualistic interactions.

Some criticism has also been made to the concept of diffuse coevolution because it seems to suggest that these communities are intractable to analysis. To get a real view of how coevolutionary interactions are shaped in species-rich communities, one has first to look at the structure of such complex networks. To do this, one needs appropriate techniques, as the ones provided by the physics of complex networks and other concepts previously applied to food web studies and that have gone through completely independent paths. If there is no structure, that is, plants and animals interact randomly, then a concept such as diffuse coevolution may be enough to describe coevolutionary interactions. If there is structure, however, the shape of this structure will certainly lead to an alternative view of coevolution.

In this chapter we will first review the search for structure in food webs. Then, we will turn to mutualistic networks, specifically to recent results showing that they are neither randomly organized nor organized in compartments; instead, they show a very cohesive structure organized in a nested, ChineseBox fashion. Second, we will discuss the consequences of this nested pattern for the coevolutionary process, community dynamics, and conservation biology. This pattern will also be related to the previous studies on food webs. We will end up by listing a series of problems that have to be solved before more progress is made towards a comprehensive description of structure in ecological networks. 


\subsection{FOOD WEB STRUCTURE}

The search for structure has a large tradition in food web research. One of the main research agendas in food webs has looked for compartments, which has largely influenced our view of community ecology (Paine 1963, May 1972). A structure in compartments or modules implies species interacting strongly within a compartment, with almost no interactions between compartments. Two approaches to food web research have produced somehow opposite results. First, studies on quantitative food webs (i.e., looking at interaction strength) have emphasized compartments (Paine 1963, 1966). The compartmentalization agenda was also largely imposed by the seminal paper by Robert M. May (1972) on the relationship between stability and complexity in food webs. At the end of the paper, May noticed that model communities tended to be more stable if organized in blocks: "our model multi-species communities, for given average interaction strength and web connectance, will do better if the interactions tend to be arranged in blocks -again a feature observed in many natural ecosystems." In here there are two important messages. First, the result that compartmentalized communities are more stable, which follows from Gardner and Ashby (1970). Second, the suggestion that real communities are structured in compartments.

The above result was highly influential and spurred a sequence of papers on the subject. Pimm (1979a), for example, looked at theoretical communities assembled under more realistic rules, that is, excluding many of the unreasonable properties found in random communities such as predators with nothing to feed on. He concluded that compartmentalization, far from stabilizing the community, destabilizes it. The debate revolved around the consequences of compartmentalization. Alternatively, papers looked at real data using qualitative food webs (interactions are not weighted by a strength). These papers were aimed at addressing whether communities are compartmentalized, but results were mainly negative (e.g., Pimm and Lawton 1980), although there are exceptions (e.g., Krause et al. 2003). Thus, compartments entered the food web research more as a consequence of Paine's and May's influential papers than as a result of empirical qualitative evidence.

An alternative view of food webs has recently emerged after the analysis of the largest food webs using new techniques such as the connectivity correlation (Melián and Bascompte 2002) and the structure of subwebs (Melián and Bascompte 2004). The former technique, derived from studies on complex networks such as the Internet (Maslov and Sneppen 2002, Pastor-Satorras et al. 2001), looks at the relationship between the number of connections of one species and the average number of connections of the species it interacts with. For example, protein networks show a negative connectivity correlation so that highly connected proteins tend to interact with weakly connected proteins (Maslov and Sneppen 2002). The dynamic consequence of this structure in compartments is to enhance the robustness of these networks to the propagation of deleterious mutations, which tend to be confined within a module. On the contrary, food 
webs suggest a non-random structure in which generalist species tend to interact among themselves (Melián and Bascompte 2002), and where a collection of small subwebs is connected to a single, highly dense subweb which glues the web altogether (Melián and Bascompte 2004). The resulting view is very cohesive. This cohesiveness would make the food webs more sensitive to the spread of a contaminant, but more robust to the loss of species.

Thus, the latter results on food webs rise the question on whether food webs are more cohesive (and so less compartmentalized) than expected. It could be interesting to analyze both qualitative and quantitative data sets to test if compartments or cohesion are essential features of food webs. A previous study has shown that ignoring weights when aggregating taxa decreases the number of analyzed interactions and can obscure strong relationships that contribute to compartmentalization (e.g., Krause et al. 2003), but more synthetic work is needed here.

Let's now turn again to mutualistic networks, the focus of our chapter. We will look at structure there and how to relate this structure to the one observed in food webs.

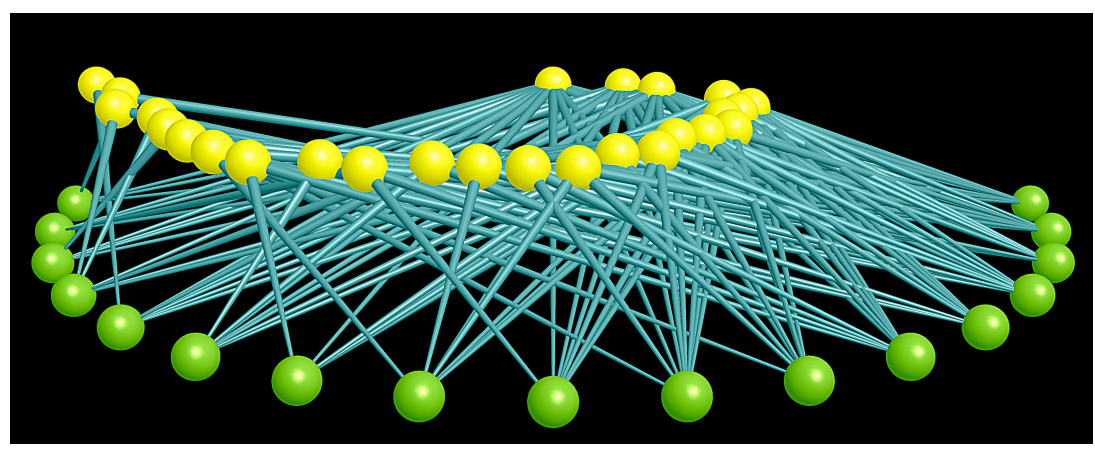

Figure 1. A plant-pollinator mutualistic network. Plants are represented as green nodes, insects as yellow nodes. This web, compiled by J.M. Olesen and H. Elberling, corresponds to the interactions reported in a single day in an Arctic community in Greenland. Courtesy of J. Olesen, plotted using the FoodWeb3D software by R. Williams. 


\section{THE NESTED STRUCTURE OF MUTUALISTIC NETWORKS}

There is a marked difference between traditional food webs and mutualistic networks. While traditional food webs are represented as undirected graphs (Cohen 1978, Cohen et al. 1990, Pimm 1982), that is, relations depicting who-eats-whom through several trophic levels, mutualistic networks are described by means of bipartite graphs (Jordano et al. 2003). Bipartite graphs depict the relationships between (but not within) two distinct sets: plants and animals. Fig. 1 represents an example of such a network. Alternatively, mutualistic networks can be described as a matrix, with plants as rows and animals as columns ${ }^{1}$ (Fig. 2). Each element of the matrix is 1 if that particular plant and animal interact, and 0 otherwise. In this chapter we will only review qualitative information, although each element of this matrix could also describe the strength of the interaction (i.e., the relative frequency of visits, or relative frequency of pollen or seeds dispersed). Plant-animal networks are an adequate way to represent a wide range of situations in which plants and animals coevolve. Some of these examples are more resolved than traditional food webs: one deals with taxonomic species. This and the fact that only two trophic levels are involved, makes of these plant-animal networks a good candidate to study organization. Recently, several researchers have focussed on the statistical properties of plant-animal networks (Jordano 1987, Memmott 1999, Memmott and Waser 2002, Vázquez and Aizen 2003, 2004, Jordano et al. 2003, Bascompte et al. 2003). This recent explosion of research has been in part spurred by work on the statistical mechanics of complex networks (Amaral et al. 2000, Albert and Barabási 2002). Particularly relevant for the discussion in here, mutualistic networks have been found to be highly nested (Bascompte et al. 2003). Nestedness is a concept borrowed from island biogeography to illustrate how a pool of animals is redistributed among a set of islands (Atmar and Paterson 1993). One can use an analogy and imagine that a plant is an "island" that harbors several animal species which feed on it.

A mutualistic matrix is nested if generalists interact with proper subsets of the set of species generalist interact with. That is, if we move along an axis from the most specialists to the most generalist, we found that the same group of species is repeated within larger groups (see Fig. 2a and 2d). Using an appropriate null model in which the probability of an interaction is proportional to both plant and animal degrees, Bascompte et al. (2003) showed that mutualistic networks are significantly nested. Thus, these networks can be described neither as random collections of interacting species, nor as compartments arising from tight, parallel specialization.

Nestedness implies two properties of these networks related to research on food webs. First, a nested matrix is highly cohesive, because generalist plants and generalist animals interact among themselves. This creates a core in which a

\footnotetext{
${ }^{1}$ the alternative, transposed arrangement with animals as rows and plants as columns is also frequently used
} 
small set of species leads the bulk of interactions. Second, these matrices embed asymmetric interactions in the sense that specialist species tend to interact with the most generalist species (both for plants and animals). Vázquez and Aizen (2004) have also detected this asymmetric pattern of specialization. In the following sections we will explore the consequences of this patter for coevolution, community dynamics, and conservation biology.
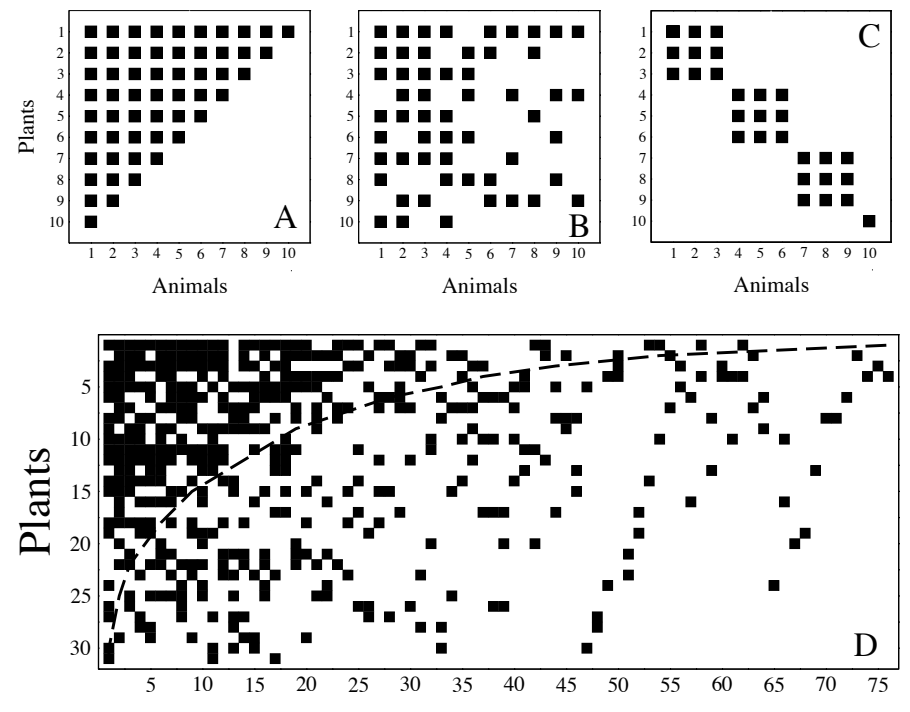

Animals

Figure 2. A to $C$ represent examples of plant-animal matrices perfectly nested, random, and organized in compartments, respectively. D represents the pollinator community plotted in the previous figure (data now correspond to cumulative interactions over the whole flowering season). This community is significantly nested.

Traditional methods to detect compartments in food webs assume that species within a compartment would share more predators and preys that they would with species outside that compartment (Pimm and Lawton 1980, Raffaelli and Hall 1992). Thus, one can calculate the degree of trophic similarity among any two pair of species (defined as the number of common species they interact with over the total number of species either one or the other interacts with, Pimm and Lawton 1980). More recently, a method for detecting compartments originated from the field of social networking has been used in food webs (Krause et al. 2003). Basically, the method is based on a comparison of the density of interactions within versus between compartments, and uses randomization procedures to test the statistical significance of that comparison (see Krause et al. for details). 
In relation to Nestedness, the degree of nestedness is calculated using the Nestedness Calculator (AICS Research, University Park, NM; Atmar and Patterson 1993). This software works with a presence-absence matrix and starts by rearranging rows and columns from the most generalists to the most specialist in a combined way that maximizes nestedness. The software calculates an isocline of perfect nestedness, which is the theoretical arrangement of rows, columns, and interactions giving place to a perfectly nested matrix. Then, it detects for each row all the unexpected present and absent interactions in the real matrix. That is, it considers the gaps in the left-hand side semimatrix (all interactions should lay in here in the perfectly nested scenario), and the observed interactions in the right-hand side semimatrix (which should be empty in the perfectly nested scenario). For each of these unexpected presences and absences, a normalized distance to the isocline is calculated, and these values are averaged. This global measure is called temperature using an analogy with physical disorder (a completely nested matrix represents order, and a random one represents disorder). Temperature $(T)$ ranges between 0 and 100 degrees. Nestednes $(N)$ can be defined as $N=(100-T) / 100$, so its value ranges between 0 and 1 .

The next step after calculating a measure of structure such as nestedness, is to use a null model as a benchmark to asses the significance of the pattern, that is, the chances of getting a similar pattern just by chance. the Nestedness Calculator uses a null model in which each cell of the matrix has the same probability of being occupied, a probability estimated as the fraction of total interactions. However, imagine that we find significant departure. This can be due to multiple reasons. If, for example, we are interested in studying the heterogeneous degree across species, that is, the variance in the number of interactions per species, the previous would be an adequate null model. Since we are testing whether we can get by chance a certain distribution of links per species given a total number of links, plants, and animals. However, if we are interested in higher levels of structure as nestedness, we can not conclude that a community is significantly nested, because departure from the previous null model could just be due to heterogeneous degree, not to nestedness. For more details about this subject see Cook and Quinn (1998), Gotelli (2001), Fischer and Lindenmayer (2002), and Vázquez and Aizen (2003) among many others. When looking for an appropriate null model we have to clearly identify at what level is our question posed. 
The nested pattern in plant-animal mutualisms is opposed to the compartmented view of food webs. This, of course, does not mean that compartments can not be found in mutualistic networks (see Dicks et al. 2002), but overall nestedness seems a more robust pattern. To asses this, however, one needs a comprehensive approach looking simultaneously for different patterns (see last section). On the other hand, nestedness is compatible with the latest results on food webs reviewed in the previous section which point out their very cohesive structure.

When looking at nestedness, one can also find cases in which a community is significantly less nested than a random one. This occurred in four cases out of 66 studied by Bascompte et al. (2003), all four corresponding to parasitoid food webs: seed feeder miner-parasitoids in Silwood Park (Memmott et al. 2000), and grass-herbivores, insect-parasitoids, and parasitoid-hiperparasitoids in Grass Stems, U.K. (Martinez et al. 1999). Parasitoids tend to be very specialist and organized in well-defined compartments (but see Memmott et al. 1994). Thus a compartmentalized plant-animal matrix would be detected as well by the nestedness analysis. However, we do not intend to say that this is the best way to look at compartments (see last section).

\section{IMPLICATIONS OF NESTEDNESS}

\subsection{IMPLICATIONS FOR COEVOLUTION}

The nested structure found in mutualistic networks has clear implications for our understanding of the coevolutionary process. As noted above, nestedness implies two relevant properties of plant-animal networks. First, the nested matrix has a dense core of species. This core is formed by the interactions among generalist plants and animals. It may be formed by a small number of species, but it contains a large fraction of the total number of interactions. This supports John Thompson's view of coevolutionary vortices, i.e., a subset of taxa within a given, highly diversified community whose interactions might "drive" the evolution of the whole system. At any community, the identity of the species forming the core is going to highly determine the selective forces exerted on other, more specialist species which will become attached to such a core. Interestingly enough, this core may change geographically, and this represents a clear link between the two major theories bringing tractability to multispecific coevolutionary studies: network theory and the geographic mosaic theory (Thompson 1994, in press).

The geographic mosaic of coevolution introduces a landscape approach to coevolution. Populations are distributed in discrete patches, and the sign of an interaction may depend on the presence in that patch of other species. The best known example is Greya Politella a moth that oviposites inside the ovary of Litophragma parviflorum, a widely distributed plant in western North America. Developing Greya larvae eat on the average between 15-27\% of the seeds of an attacked flower. This is an example of antagonism, since it reduces the plant's 
fitness. However, it is a low reduction, and interestingly enough, since Greya oviposites in several flowers, also acts as a mutualism by pollinating Litophragma (Thompson 1994). The net sign of the interaction depends on whether there are other co-pollinators in the area. Across the distribution of Litophragma there are areas in which the interaction is of a mutualistic type, others where is antagonistic, and still others in which both effects balance out. The important point in here is that the landscape approach brings new ingredients to understand such a plant-animal interaction. For example, at some patches there may be mismatches in the adaptation between both species which can only be accounted for if one looks at a broad geographic scale: other patches act as coevolutionary hotspots maintaining such interactions (Thompson 1994).

The geographic mosaic of coevolution is also a theory on plant-animal interactions which can be described neither as pair-wise nor diffuse. While the geographic mosaic puts the emphasis on the landscape component, the network approach emphasizes the highly structured organization of interactions within communities. What would be interesting is to bridge between both approaches. This is quite natural if one keeps in mind that nestedness was first introduced into the realm of island biogeography (Atmar and Patterson 1993). This is equivalent to look at the component of local structure explained by landscape-level properties. For example: are the more widespread species also the more generalist and so the ones occupying the core of the mutualistic matrix? Or, on the contrary, if there is no relationship between spatial and network structure, is the core of species highly variable among local communities?

A second implication for coevolution is that network structure complements one of the long-standing debates in mutualistic studies: the specialization-generalization debate (e.g. Waser et al. 1996). There have been lots of discussions about whether specialist interactions are the rule or the exception, or in other words, about the widespread presence of generalism in mutualistic communities. In a highly influential paper, Waser et al. (1996) showed that the bulk of interactions are generalists, and explored the ecological implications of this finding. As Vázquez and Aizen (2003) have correctly stated, even when talking about specialization-generalization, we have to use a null model as a benchmark, because the key question is not whether we observe specialization, but whether the level of specialization we observe is higher than the one we would observe just by chance. Vázquez and Aizen concluded that the number of highly specialist and generalist species is significantly higher than the value expected under a simple null model based on a similar probability of interaction across species. However, when controlling for differences across species in the frequency of interactions, the differences disappeared, that is, there is neither more generalization nor more specialization that what we would expect under these premises. An important conclusion of Vázquez and Aizen's (2003) paper is that they identify a close relationship between the degree of generalization of a species and their frequency of interactions, which may bring insight into the mechanisms leading to the observed structure. 
The network approach brings a new dimension to the generalization-specialization debate. The focus now is as follows: given a certain distribution of specialists and generalists, how are they related? As noted, nestedness implies that generalist plants and animals interact among themselves more than expected by chance, and that specialists tend to interact with generalists. In addition, the network approach shows clearly that certain properties of highly diversified mutualisms, such as asymmetry, cannot be understood without a communitylevel approach; understanding specialization-generalization at the species level is insufficient.

\subsection{IMPLICATIONS FOR COMMUNITY DYNAMICS}

It is well-known that structure greatly affects dynamics. This spurred the search for compartments in food webs. There have been two main approaches to food web dynamics: demographic and topologic stability. Demographic stability is based on the study of local stability in the vicinity of a steady state. It describes whether small fluctuations around this steady state will amplify or die out through time (May 1973). On the other hand, topological stability describes species as nodes of a network, and looks at the effects for network connectivity of removing some nodes. This follows the influential work by Albert et al. (2000) on the Internet, although species deletion stability had already been explored in ecology by Pimm (1979b) using simulations, and by Paine (1980) and others using an experimental approach.

Recently, the complex network approach has produced a series of papers looking at how food webs respond to the loss of species (both randomly chosen or chosen according to their number of links, Albert et al. 2000, Solé and Montoya 2001, Dunne et al. 2002, Jordano et al., in press). From these studies, it is well known that the pattern of connectivity distribution affects robustness. In this paper we have looked at a deeper level of structure beyond the degree distribution. One can argue that a cohesive pattern such as nestedness is likely going to make these networks more robust to the loss at random of species or connections. Other things being equal, a cohesive network has alternative states, it is more redundant, and so it will not get fragmented as easily. The core of a nested matrix can be understood from the point of view of percolation theory. In a nested matrix, the density of interactions in the core is largely beyond the percolation threshold, which means that one can find a path from one side to another of the core following observed interactions. Stability, however, has several sides, and a cohesive pattern, even when may reduce the risk of network fragmentation, is more susceptible to the spread of a contaminant trough the network or to the invasion of a new species into the community (Melián and Bascompte 2002). However, more studies are necessary, and particularly studies relating dynamic and topologic approaches to stability.

Also, the nested structure of plant-animal mutualisms implies that rare, specialist species tend to interact with generalists. Other things being equal, 
generalist tend to be more abundant and less fluctuating since they rely on multiple resources (e.g., Turchin and Hanski 1997). Thus, the nested structure provides pathways for the persistence of rare species.

\subsection{IMPLICATIONS FOR CONSERVATION BIOLOGY}

The network approach to mutualistic interactions here reviewed has clear implications for management. An expanded concept of biodiversity has to address the interactions among species besides species themselves. Interactions are the "glue of biodiversity" (Thomson 2004). One example of how the network approach can be useful in the context of conservation biology is ecosystem restoration, a subject covered in another chapter by Jane Memmott. Conservation biology has been oftentimes too narrowly focused on the target species, but thinking at larger scales may be very important. Imagine we want to preserve an endangered insect species. We certainly have to make sure that the plants it relies on are present in the area. However, because of the asymmetry in the interactions arising from the nested structure, these plants will depend largely on other insects which are not the target taxa for conservation. Without ensuring the visits on these latter species, the plants may decrease in abundance and push the target insect towards extinction. Knowing that mutualistic networks are nested may help in planning these strategies.

An increasing problem on tropical ecosystems is defaunation (Dirzo and Miranda 1990). Hunting preferentially affects large mammals and birds that are keyspecies in relation to seed dispersal. These species are highly mobile, and so account for the episodes of long-distance dispersal. These episodes, even when not very common, have a huge importance for gene flow (Clarke et al. 1990). The problem is larger than expected, because woody plants may be very longeve, and are still there for us to see them. However, they may be ecological ghosts since without megafauna, their seeds are wasted beneath the mother tree (Cordeiro and Howe 2003). To assess the community-wide importance of the loss of megafauna we have to relate the nested structure of mutualistic networks with ecological correlates. For example, are large-bodied frugivorous randomly scattered through the matrix of interactions, or do they tend to be the generalists forming the core? If the second, the nested structure implies that loosing these few species may lead to a collapse of the network.

Another important issue is related to the propagation of infectious diseases among insects via host plants which are pollinated. This is similar to the role of network topology in sexually transmitted diseases in humans (Liljeros et al. 2001, Pastor-Satorras and Vespigniani 2001). If a disease or a parasite can be transmitted across several insect species, the structure of the network of interactions is largely going to affect the propagation of the disease. For example, theoretical work on computer viruses has shown that the classical eradication thresholds observed in epidemiological models assuming a random network of interactions disappear when the topology of the network of interactions follows a power-law 
distribution (i.e., it is highly skewed so that a small fraction of individuals have a huge number of interactions, Pastor-Satorras and Vespigniani 2001). This line of research should be extended to look at deeper levels of structure such as the ones discussed in here. For example, for a specific connectivity distribution, which network structure reduces the chance of disease propagation? If the networks is compartmentalized, the effects will be contained within a compartment. If, on the other hand, is highly nested, it will propagate easily throughout the network.

Network structure is also related to the likelihood of biological invasions. The invasion of exotic species is one of the leading factors in biodiversity decline after habitat destruction. Increases in commerce and travel have lead to an increase of exotic species with fatal consequences for native plants and animals (Liebhold et al. 1995, Vitousek et al. 1996, Simberloff 2001). In plant communities, the likelihood of an invasion declines as species richness increases (Kinzig et al. 2002). This clearly illustrates how important are characteristics at the level of the target community. What remains to be studied is whether, for a specific number of species, the way these are connected also affects the likelihood of an invasion. Memmott and Waser (2002) have looked at the integration of alien plants and insects into a pollination network. They concluded that flowers of alien plants were visited by less animal species than flowers of native plants. Also, visitors to alien plants tend to have exceptionally broad diets. This is in agreement with the predictions of a nested community. If alien plants are on average more specialist, the prediction is that they will tend to interact with generalists as noted above. This tendency, other things being equal, provides aliens with a more abundant and reliable resource. The question now is whether native communities are more nested than communities with a large fraction of exotics. If so, dynamic differences between these two communities can be directly inferred. Similarly, when we look at the species level, it would be interesting to compare between native and exotic species in the degree they contribute to decrease the level of nestedness of the community. If, for example, exotic species on average tend to decrease the level of nestedness, then their arrival to that community will modify community-wide coevolutionary and stability properties.

Understanding the nested structure of mutualistic networks can explain why pollination generalist and specialist plant species are similarly affected by habitat fragmentation (Ashworth et al., 2004). In principle, one would assume that specialist plants are more vulnerable to this destructive process since any significant decrease in the abundance of its only pollinator would lead to failure in reproduction. However, Aizen et al. (2002) found that a similar fraction of generalist and specialist plant species were affected by habitat fragmentation. This can be explained by the asymmetry in the interactions (specialists tend to interact with generalists) arising from a nested structure. Specifically, generalist plants interact with lots of specialist pollinators, which would go extinct soon, thus leaving the plant with a few generalist pollinators, a situation similar to the one experienced by specialists plants which retain their few generalist pollinators until moderate to high values of habitat destruction (Ashworth et al. 
2002). Therefore, when assessing the effects of habitat fragmentation, we should not consider the level of generalization of plant species in isolation, but in combination to the level of generalization of its pollinators. In other words, it is a combined property of plants and animals which is relevant to asses the consequences of habitat loss and similar perturbations. Again, from the above one can infer that the nested assembly of plant-animal mutualistic interactions provides mechanisms for the persistence of specialist species.

\section{CHALLENGES}

The next stage in the search for structure in plant-animal assemblages is to compare both antagonistic and mutualistic networks. Preliminary studies on plantherbivore interactions seem to suggest that in this case the structure can be better described by compartments (Prado and Lewinsohn in press, Lewinsohn et al., submitted). However, in order to proceed towards a rigorous comparison across different systems, we need more data on plant-animal antagonistic networks. If a difference exists as these preliminary results seem to suggest, one could explore the different mechanism acting on these two types of networks to create different structures.

One particular problem is that studies look only for a particular type of structure, either compartments or nestedness. Finding evidence for a particular type of structure does not preclude other structures (Leibold and Mikkelson 2002, Lewinsohn et al., submitted). Thus, in order to make a serious inference about structure we need a more comprehensive approach in which one devises methods that simultaneously look for different structures and detect the one that better describes the data. One recently developed approach is based on the study of eigenvalues of properly rearranged matrices (Lewinsohn et al., submitted).

Another serious problem concerns the comparison between plant-animal interaction networks and food webs. As noted above, these are two completely different types of networks from an analytical point of view (Jordano et al., in press). In order to make them comparable, we have to transform them into a common format. This can be the niche overlap graph (sensu Cohen 1978) where two species in a food web are linked if they share a common prey. Similarly, we would have two niche overlap matrices in plant-animal interactions: one relating two animals if they pollinate or disperse at least a common plant, and the equivalent for the plants (i.e., two plants will be related if they share at least a common pollinator). The task is to see whether measures of structure in original matrices and transformed matrices are related, and to compare measures of structure in transformed matrices for food webs, plant-animal, and other types of networks (Olesen et al., in preparation). Only then, we will be able to infer what is common and what is different in the architecture of networks from different types of interactions. 


\section{ACKNOWLEDGMENTS}

This paper has been supported by the Spanish Ministry of Education and Science (Grants REN2003-04774 to JB and REN2003-00273 to PJ). We thank C.J. Melián, M.A. Fortuna, J.M. Olesen, John Thompson and T. Lewinsohn for interesting discussions. 


\section{References}

[1] Aizen, M.A., L. Ashworth, and L. Galetto. "Reproductive Success in fragmented Habitats: do Compatibility Systems and Pollination Specialization Matter?" J. Veget. Sci. 13 (2002): 885-892.

[2] Albert, R., H. Jeong, and A.-L. Barabási. "Error and Attack Tolerance of Complex Networks." Nature. 406 (2000): 378-382.

[3] Albert, R., and A.L. Barabási. "Statistical Mechanics of Complex Networks." Rev. Modern Phys. 74 (2002): 47-97.

[4] Amaral, L.A., A. Scala, M. Barthelemy, and H.E. Stanley. "Classes of SmallWorld Networks." Proc. Natl. Acad. Sci USA 97 (2000): 11149-11152.

[5] Ashworth, L., R. Aguilar, L. Galetto, and M.A. Aizen. "Why Do Pollination Generalist and Specialist Plant Species Show Similar Reproductive Susceptibility to Habitat Fragmentation?" J. Anim. Ecol. 92 (2004): 717-719.

[6] Atmar, W., and B.D. Patterson. "The measure of Order and Disorder in the Distribution of Species in Fragmented Habitat." Oecologa. 96 (1993).

[7] Bascompte, J., P. Jordano, C.J. Melián and J.M. Olesen. "The Nested Assembly of Plant-Animal Mutualistic Networks." Proc. Natl. Acad. Sci. USA 100 (2003): 9383-9387.

[8] Clarke, J.S., M. Silman, R. Kern, E. Macklin, and J. Hilleris-Lambers. "Seed dispersal near and far: Patterns across temperate and tropical forests." Ecol- 
ogy 80 (1999): 1475-1494.

[9] Cohen, J.E. Food Webs and Niche Space. Princeton, USA: Princeton University Press, 1978.

[10] Cohen, J.E., F. Briand, and C.M. Newman Community Food Webs: Data and Theory. Berlin, Germany: Springer-Verlag, 1990.

[11] Cook, R.R., and J.F. Quinn. "An Evaluation of Randomization Models for Nested Species Subsets Analysis." Oecologia. 113 (1998): 584-592.

[12] Cordeiro, N.J., and H.F. Howe. "Forest Fragmentation Severs Mutualism Between Seed Dispersers and an Endemic African Tree." Proc. Natl. Acad. Sci. USA 100 (2003): 14052-14056.

[13] Dicks, L.V., S.A. Corbet, and R.F. Pywell. "Compartmentalization in PlantInsect Flower Visitor Webs." J. Anim. Ecol. 71 (2002): 32-43.

[14] Dirzo, R., and A. Miranda. "Contemporary Neotropical Defaunation and Forest Structure, Function and Diversity." Cons. Biol. 4 (1990): 444-447.

[15] Dunne, J.A., R.J. Williams, and N.D. Martinez. "Network Structure and Biodiversity Loss in Food Webs." Ecol. Lett. 5 (2002): 558-567.

[16] Ehrlich, P.R., and P.H. Raven. "Butterflies and Plants: a Study in Coevolution." Evolution 54 (1964): 1480-1492.

[17] Fisher, J., and B. Lindenmayer. "Treating the Nestedness Temperature Calculator as a Black Box can Lead to False Conclusions." Oikos. 99 (2002): 193-199.

[18] Gardner, R.M., and W.R. Ashby. "Connectance of Large Dynamic (Cybernetic) Systems: Critical Value of Stability." Nature 228 (1970): 784.

[19] Gotelli, N.J. "Research Frontiers in Null Models." Global Ecol. Biog. 10 (2001): 337-343.

[20] Herrera, C.M. "Variation in Mutualisms: the Spatio-Temporal Mosaic of a Pollinator Assemblage." Biol. J. Lin. Soc 35 (1988): 92-125.

[21] Janzen, D.H. "When is it Coevolution?" Evolution 34 (1980): 611-612.

[22] Jordano, P. "Patterns of Mutualistic Interactions in Pollination and Seed Dispersal: connectance, Dependence Asymmetries, and Coevolution." Am. Nat. 129 (1987): 657-677.

[23] Jordano, P. "Fruits and frugivory." in M. Fenner, ed. Seeds. the Ecology of Regeneration in Plant Communities, 125-166. Wallingford, U.K.: CABI Publ., 2000.

[24] Jordano, P., J. Bascompte, and J. M. Olesen. "Invariant Properties in Coevolutionay Networks of Plant-Animal Interactions." Ecol. Lett. 6 (2003): 69-81.

[25] Jordano, P., J. Bascompte, and J.M. Olesen. "The Ecological Consequences of Complex Topology and Nested Structure in Pollination Webs." in N. Waser and J. Ollerton, eds. Specialization and Generalization in PlantPollinator Interactions, Chicago, USA: Chicago University Press, in press.

[26] Kinzig, A.P., S. Pacala, and D. Tilman, Eds. The Functional Consequences of Biodiversity: Empirical Progress and Theoretical Extensions. Princeton, USA: Princeton University Press, 2002. 
[27] Koh, L.P., R.R. Dunn, N.S. Sodhi, R.K. Colwell, H.C. Proctor, and V.S. Smith. "Species Coextinctions and the Biodiversity Crisis" Science 305 (2004): 1632-1634.

[28] Krause, A.E., K.A. Frank, D.M. Mason, R.E. Ulanowicz, and W.W. Taylor. "Compartments Revealed in Food-Web Structure" Nature 426 (2003): 282285.

[29] Leibold, M.A., and G.M. Mikkelson. "Coherence, species turnover, and boundary clumping: elements of meta-community structure." Oikos $\mathbf{9 7}$ (2002): 237-250.

[30] Lewinsohn, T.M., P. Jordano, P.I. Prado, J.M. Olesen, and J. Bascompte. "Structure in Plant-Animal Interaction Assemblages." Submitted.

[31] Liebhold, A., W.L.D. MacDonald, D. Bergdahl, and V. Mastro. "Invasion by Exotic Forest Pests: a Threat to Forest Ecosystems." For. Sci. Mon. 30 (1995): 1-49.

[32] Liljeros, F., C.R. Edling, L.A.N. Amaral, H.E. Stanley, and Y. Aberg. Turchin, P., and I. Hanski. "The Web of Human Sexual Contacts." Nature 411 (2001): 907-908.

[33] Martinez, N.D., B.A. Hawkins, H.A. Dawah, and B.P. Feifarek. "Effects of Sample Effort on Characterization of Food-Web Structure." Ecology 80 (1999): 1044-1055.

[34] Maslov, S., and K. Sneppen. "Specificity and Stability in Topology of Protein Networks." Science 296 (2002): 910-913.

[35] May, R.M. "Will a Large Complex System be Stable?" Nature 238 (1972): 413-414.

[36] May, R.M. Stability and Complexity in Model Ecosystems. Princeton, USA: Princeton University Press, 1982.

[37] Melián, C.M., and J. Bascompte. "Complex Networks: Two Ways to be Robust?" Ecol. Lett. 5 (2002): 705-708.

[38] Melián, C.M., and J. Bascompte. "Food Web Cohesion." Ecology 85 (2004): 352-358.

[39] Memmott, J. "The Structure of a Plant-Pollinator Network." Ecol. Lett. 2 (1999): 276-280.

[40] Memmott, J., H.C.J. Godfray, and I.D. Gauld. "The Structure of a Tropical Host Parasitoid Community." J. Anim. Ecol. 63 (1994): 521-540.

[41] Memmott, J., N.D. Martinez, and J.E. Cohen. "Predators, Parasitoids and Pathogens: Species Richness, Trophic Generality and Body Sizes in a Natural Food Web." J. Anim. Ecol. 69 (2000): 1-15.

[42] Memmott, J., and N.M. Waser. "Integration of Alien Plants into a Native Flower-Pollination Visitation Web." Proc. R. Soc. London B 269 (2002): 2395-2399.

[43] Simberloff, D.S. "Eradication of Island Invasives: Practical Actions and Results Achieved." Trends Ecol. Evol. 16 (2001): 273-274.

[44] Paine, R.T. "Trophic relationships of 8 sympatric predatory gastropods." Ecology 44 (1963): 63-73. 
[45] Paine, R.T. "Food web complexity and species diversity." Am. Nat. 100 (1966): 65-75.

[46] Paine, R.T. "Food Webs: Linkage, Interaction Strength and Community Infrastructure." J. Anim. Ecol. 49 (1980): 667-685.

[47] Pastor-Satorras, R., and A. Vespigniani. "Epidemic Spreading in Scale-Free Networks." Phys. Rev. Lett. 86 (2001): 3200-3203.

[48] Pastor-Satorras, R., A. Vazquez, and A. Vespignani. "Dynamical and Correlation Properties of the Internet." Phys. Rev. Lett. 87 (2002): 258701.

[49] Pimm, S.L. "The Structure of Food Webs." Theor. Pop. Biol. 16 (1979a): 144-158.

[50] Pimm, S.L. "Complexity and Stability; Another Look at MacArthur's Original Hypothesis." Oikos 33 (1979b): 351-357.

[51] Pimm, S.L., and J.H. Lawton. "Are Food Webs Divided into Compartments?" J. Anim. Ecol. 49 (1980): 979-898.

[52] Pimm, S.L. Food Webs. London, U.K.: Chapman and Hall, 1982.

[53] Prado, P.I., and T.M. Lewinsohn. "Compartments in Insect-Plant Associations and their Consequences for Community Structure." J. Anim. Ecol., in press.

[54] Raffaelli, D., and S.J. Hall. "Compartments and Predation in an Estuarine Food Web." J. Anim. Ecol. 61 (1992): 551-560.

[55] Simberloff, D.S. "Eradication of Island Invasives: Practical Actions and Results Achieved." Trends Ecol. Evol. 16 (2001): 273-274.

[56] Solé, R.V., and J.M. Montoya. "Complexity and Fragility in Ecological Networks." Proc. R. Soc. London B. 268 (2001): 2039-2045.

[57] Thompson, J.N. The Coevolutionary Process. Chicago, USA: University of Chicago Press, 1994.

[58] Thompson, J.N. "Plant-animal interactions: future directions. " in C.M. Herrera and O. Pellmyr, eds. Plant-Animal Interactions. An Evolutionary Approach, 236-247. Oxford, U.K.: Blackwell, 2002.

[59] Thompson, J.N. The Geographic Mosaic of Coevolution. In press.

[60] Turchin, P., and I. Hanski. "An Empirically-Based Model for the Latitudinal Gradient in Vole Population Dynamics." Am. Nat. 149 (1997): 842-874.

[61] Vázquez, D.P., and M.A. Aizen. "Null Model Analysis of Specialization in Plant-Pollinator Interactions." Ecology 84 (2003): 2493-2501.

[62] Vázquez, D.P., and M.A. Aizen. "Asymmetric Specialization: a Pervasive Feature of Plant-Pollinator Interactions." Ecology 85 (2004): 1251-1257.

[63] Vitousek, P.M., C.M. D'Antonio, L.L. Lope, and R. Westbrooks. "Biological Invasions as Global Environmental Change." Am. Scientist 84 (1996): 468478.

[64] Waser, N., M.V. Price, N.M. Williams, and J. Ollerton. "Generalization in Pollination Systems and Why it Matters." Ecology 77 (1996): 1043-1060. 